\title{
Microhabitats de Aedes albopictus (Skuse) na região do Vale do Paraiba, Estado de São Paulo, Brasil*
}

\author{
Microhabitats of Aedes albopictus (Skuse) in the Paraiba Valley region of the \\ State of S. Paulo, Brazil
}

Almério de Castro Gomes**, Oswaldo Paulo Forattini**, iná Kakitani** Gisela Rita A. M. Marques"**, Cristiano C. de Azevedo Marques"**, Daniel Marucci"*, Marylene de Brito"**

\begin{abstract}
GOMES, A. de C. et al. Microhabitats de Aedes albopictus (Skuse) na região do Vale do Paraíba Estado de São Paulo, Brasil. Rev. Saúde públ., S. Paulo, 26: 108-18, 1992. Objctivou-se determinar o nível de disseminação de Ae. albopictus na região do Vale do Paraíba, Estado de São Paulo, Brasil. Foram realizadas coletas de larvas e pupas $\mathrm{cm}$ seis locais distintos, segundo transecto com $10 \mathrm{~km}$ de extensão. $\mathrm{O}$ alvo principal foi oco de árvores, tendo sido também incluídos artefatos antrópicos. As espécies Ae. terrens e Ae. albopictus foram as únicas do gêncro Aedes presentes e, algumas vezes, coexistiram no mesmo microhabitat natural. A segregação de sete espécies da comunidade de oco variou de acordo com os macro e microhabitats exaninados. Assim, a distribuição de Ae. albopictus envolveu as zonas rural, rural-urbana e urbana, porém a segunda árca foi a mais preferida. Como fator favorável à infestação de Ae. albopictus na região, destacou-se a vacância de nichos ecológicos naturais resultante da influência antrópica. As chuvas foram relcvantes no "input" de larvas e pupas e os ocos, com volumes superiores a $600 \mathrm{ml}$, foram os mais produtivos. A abundância desses dois estádios ocorreu nas estações verão-outono, sendo o pico máximo alcançado nos meses de março-abril. Essa sazonalidade foi comum em bambu experimental e recipientes artificiais. Os dados de temperatura média sugeriram a laixa de 23 a $17^{\circ} \mathrm{C}$ como o periodo mais favorável ao desenvolvimento larvário. Face a isso, a cepa de Ae. albopictus estudada parece ser oriunda da Ásia tropical e, portanto, se reveste de elevada importância epidemiológica pela possibilidade dessa espécie vir a exercer, além do dengue, papel vetorial para a febre amarcla no Brasil.
\end{abstract}

Descritores: Aedes. Ecologia de vetores.

\section{Introdução}

No que concerne às pesquisas sobre mosquito Culicidae vetores de doenças nas Américas, os cstudos dos últimos cinco anos passaram a ter do lcvar em conta a situação do Aedes albopictus. Neste breve espaço de tcmpo, esse mosquito tcm ampliado significantemente sua distribuição gcográlica, somando-se às outras importantes populaçōes vctoras de arboviroses em nosso meio (PAHO ${ }^{16}, 1987 \mathrm{e}$

* Pesquisa financiada pela Fundação de Amparo à Pesquisa do Estado de São l'aulo (FAPI:Sl' - Processon ${ }^{2}$ 89/0548-3.

* Departamento de Epidemiologia da Faculdade de Saúde Pública da Universidade de São Paulo - São Paulo, Sl Brasil.

*** Superintendência de Controle de Endcmias (SUCEN). Taubaté, SP - Brasil.

Separatas/Reprints: A. de C. Gomes - Av. Dr. Amaldo, 715 01246-904 - São P’aulo, SP - Brasil.

Publicação financiada pela TAPESP. Processo Saúde Coleliva 91/4994-0
Buralli e col. $\left.{ }^{2}, 1991\right)$. É sabido que Ae. albopictus tem origem asiálica e seu espectro de distribuição espacial cnvolve gradiente que vai da floresta à área urbana. Scus microhabitats naturais são oco de árvore, internódio de bambu e gama diversificada de recipientes artificiais (Hawley ${ }^{7}, 1988$ ) sem, contudo, tornar-se tão dependente do homem como Aedes aegypli (Moore c col. ${ }^{12}, 1988$ ).

A imigração de Ae. albopictus para o Continente amcricano tem envolvido latilude norte e sul (PAHO $\left.{ }^{16}, 1987\right)$ e scu sucesso no Brasil dependerá de adaptação às condições ecológicas peculiares daqui ou de sua habilidade em competir pelas fontes de alimento c espaço. Neste sentido, apesar da suposta expectativa de sua fixação definitiva em território brasilciro, o prognóstico dos problcmas que esta infestação possa trazcr continua sendo uma incógnita.

Observaçoes iniciais levadas a efeito no Estado de São Paulo (Brito c col. ${ }^{1}, 1986$ e Gomes e col. ${ }^{5}$, 1988 ) induzcm à suposição de que esta espécie se cncontre amplamente disseminada na região do

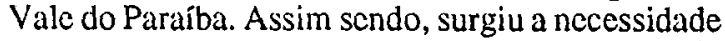
de se avaliar essa siluação, identificando as con- 
dições ambientais favoráveis à sua adaptação naquele Estado, bem como, em relação às barreiras naturais impostas à infestação por esse mosquito.

Dado que Ae. albopictus é habitante natural dos ocos das árvores na Ásia, sua presença em área urbana da região do Vale do Paraíba enseja oportunidade para saber se essa espécie terá o mesmo comportamento aqui. Assim sendo, o objetivo deste estudo dirigiu-se ao exame desses microhabitats e de artefatos antrópicos, encontrados em transecto que tem duas florestas e uma área urbana como li- mites extremos. Simultaneamente, foi observada sua colonização $\mathrm{cm}$ intcrnódios de bambus experimentais. Além da descrição dos tipos de microhabitats utilizados por essa espécie, fez-se avaliação do comportamento sazonal de larvas e pupas em relação aos ambientes e microhabitats preferidos.

\section{Região estudada}

A região do Vale do Paraíba, escolhida para a presente pesquisa, situa-se entre os dois maiores

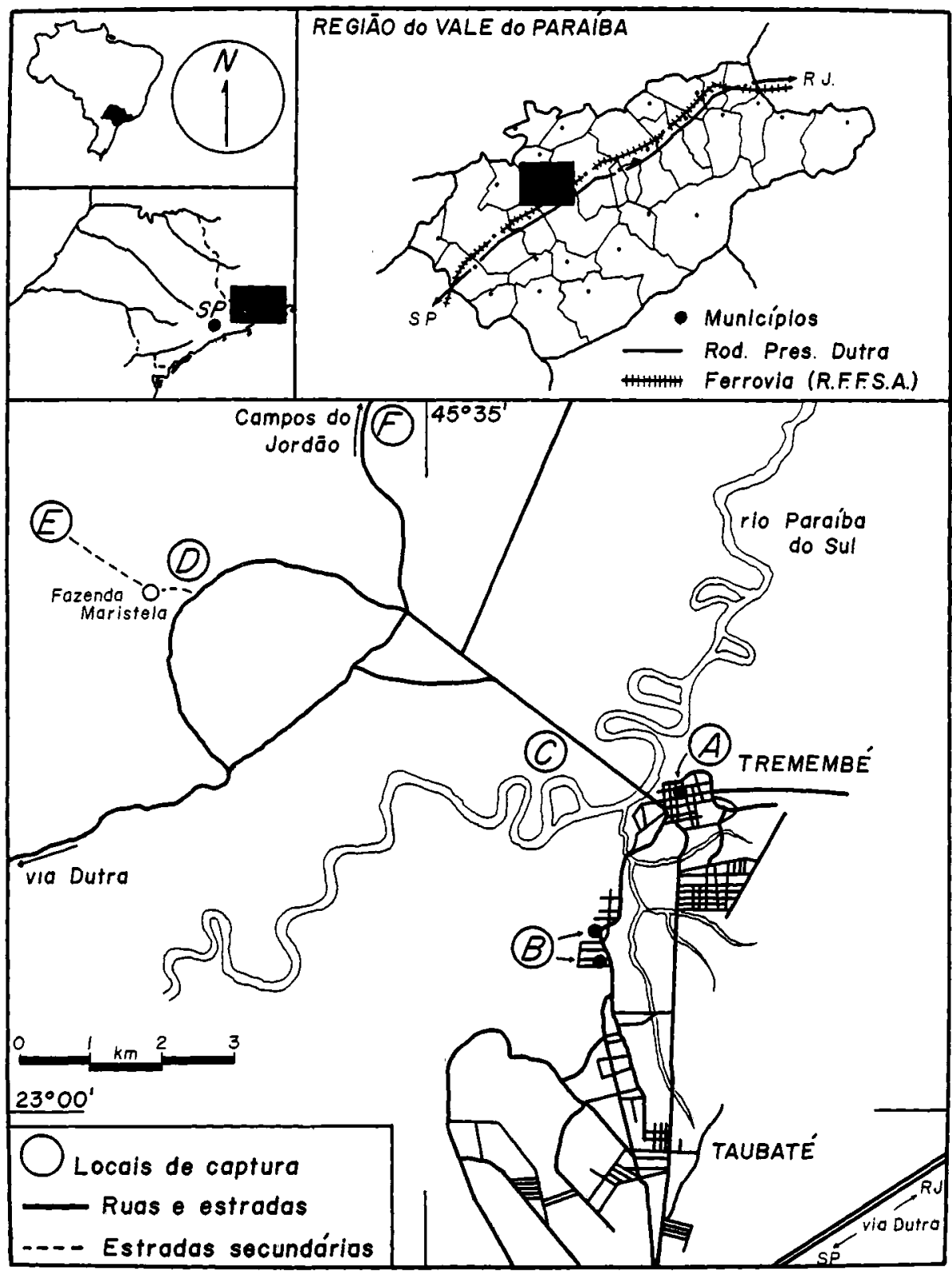

Figura 1. Distribuição das localidades investigadas no Municipio de Tremembé. A-área urbana; B-chácaras; C-mata secundária; D-alameoja; E e F mata residual primária. 
centros urbanos do Brasil, representados por São Paulo e Rio de Janeiro (Fig. 1). As interligações terrestres existentes se fazem pela Rodovia Presidente Dutra (via Dutra) c Rede Ferroviária Fedcral (RFF).

A paisagem fisiográfica da região mostra planície aluvial com altitude variando de 500-600 $\mathrm{m}$ acima do nível do mar, enquanto que a parte mais montanhosa corresponde à encosta ocidental da Serra da Mantiqueira, à região nordeste da Serra da Bocaina e, mais distante ainda, a Serra do Mar (Secretaria de Economia e Planejamento ${ }^{20}$, 1978). A cobertura florestal primária é do tipo tropical perenifólia com araucária. Sua extensão foi grandemente comprometida com as atividades agropecuárias e com a urbanização. Portanto, o que subexiste são resíduos localizados nas partes mais íngremes do terreno montanhoso. No período de $1960 / 80$ a floresta foi reduzida de $35 \%$ e a pastagem ocupava $45 \%$ da área cultivada pclo caĺé; a lavoura temporária com apenas $10 \% \mathrm{e}$, a permanente, 2,5\% (Marangone e Ortiz ${ }^{9}, 1985$ ). Há registro também de aumento da mata secundária dcvido ao reflorestamento, sendo que, de 15.005 ha em 1950 passou para 62.083 ha cm 1980. Alćm disso, destaca-se a ocorrência comum de arborização das cidades com Delonix regia (Ilambuaiã).

O clima é mesotérmico, de inverno seco com temperatura média variando bastante em função das influências topográficas. Os meses mais frios são junho e julho, com média inferior a $18 \mathrm{C}$ e os mais quentes, janeiro e fevereiro, com média supcrior a $22^{\circ} \mathrm{C}$. A distribuição das chuvas guarda relação também com o rclevo. Assim sendo, as médias anuais da vertente occânica está em torno de $2.500 \mathrm{~mm}$; da escarpa da Mantiqueira $2.000 \mathrm{~mm}$; da Serra da Bocaina $1.900 \mathrm{~mm}$ e na planície de $1.500 \mathrm{~mm}$. Os períodos secos e úmidos altcrnados ocorrem respectivamente de abril a setembro c de oulubro a março (Secretaria de Economia e Plancjamento ${ }^{20}$, 1978).

Da região do Vale do Paraíba foi escolhida, para o presente estudo, a localidade de Tremembé, situada a $22^{\circ} 57^{\prime}$ de latitude sul e $45^{\circ} 33^{\prime}$ de longitude oeste (Fig. 1). A taxa de urbanização do município é de $80 \%$ e, como conseqüência, jă existe conurbação Tremembé/Taubaté, com a inclusão, em breve, de Pindamonhangaba. Mesmo assim, chácaras c sílios são comuns no município, os quais estão scrvidos por rede de eletrificação e de abastecimento de água. No presente estudo, este ambiente foi considerado como sendo zona rural-urbana, enquanto que a rural correspondeu aos locais mais afastados onde predomina a criação de gado ou pastagem.

Os locais fixos de coletas foram distribuídos em transecto de aproximadamente $10 \mathrm{~km}$ de $\mathrm{cx}$ tensão (Fig. 1). Cada um dos pontos investigados foram os seguintes:

Local A - Foi cscolhida faixa da cidade de Tre- membé com cerca de $700 \mathrm{~m}$ de extensão. Foram investigados o peridomicílio de oito habitações de padrão social elevado; um terreno utilizado como garagem dos veículos da prefeitura local e uma praça bem arborizada. Todas as residências possuíam grandes quintais contendo árvores que produziam sombras (Fig. 1).

Local B - Duas chácaras com dimensões de 3.750 e $1.500 \mathrm{~m}^{2}$ foram escolhidas como representantes da área rural-urbana. Uma das chácaras esLava habitada, enquanto a outra estava completamente abandonada. A vegetação dessas chácaras formava conjunto com formação de extensas sombras. Os limites adjacentes latcrais também eram chácaras com características semelhantes (Fig. 1).

Local C - A escolha recaiu sobre mata secundária com dimensões de 3 ha e localizada próximo à margem do rio Paraíba (Figs. 1 e 3C). Este local estava circundado por pastagens e várias olarias muito primitivas. Os indivíduos arbóreos cram de pequeno e médio porte, e várias habitaçōes humanas formavam a paisagem domiciliar.

Local D - Um conjunto de árvores de médio porte (Cassia) formava alameda de acesso a um hotel fazenda. Os terrenos laterais à alameda serviam como pastagem para bovinos e eqüinos, nos quais haviam dois estábulos e um lago abandonado (Figs. 1e 3D).

Local E - Uma mata primária residual distando apenas $2,5 \mathrm{~km}$ do hotel fazenda acima referido foi utilizada como um dos pontos de observaçōes silvestres do estudo. Isolada c com ondulações que produziam declives de várias intensidades, as árvores eram representantes da Mora primária nativa da região. Sua extensão era de 42,3 ha (Figs. 1 e 3E).

Local F - Este foi o ponto mais distante de Centro urbano da cidade de Tremembé. Constituilu-se em mata primária com área de 68 ha e siluada à margem da rodovia SP 123 que liga Campos do Jordão ao Municipio de Tremembé e via Dutra (Figs. $1 \mathrm{e} 3 \mathrm{~F}$ ). Semelhantemente à mata anterior, apresentava declives variáveis e poucas altcraçōes. A presença de representantes de araucária e outras árvores de grande porte, formava a cobertura florestal desse local.

Quanto aos antecedentes que justificaram a escolha do Vale do Paraiba para sediar o presente estudo, estão o registro de presença de Ae. albopictus, envolvendo 32 municípios da regiāo, e 610 casos suspeitos de dengue não autóctones, com 85 deles confirmados entre 1987 e primciro trimestre de 1991. Tais informações estão registradas na Superintendência de Controle de Endemias (SUCEN)-Regional Taubaté. Além disso, a infestação domiciliar já atingiu 20 municípios, sendo que $\mathrm{cm}$ 12 a taxa de urbanização é supcrior a $80 \%$ e sobrepõem ao cixo da Via Dutra e RFF. 


\section{Material e Método}

O procedimento sistemático das observações implicou a escolha dos ocos das árvores em todos os locais anteriormente mencionados. Nessa oportunidade foi determinada a capacidade volumétrica e a profundidade individual de cada oco, colocando água destilada no oco até o limite máximo permitido, seguido da introdução de régua até atingir a parte mais profunda. A relirada completa da água dava a medida do volume máximo de cada oco e a régua a profundidade. A localização dos ocos estudados distribuiu-se preferencialmente na região correspondente ao ecótono $\mathrm{e} \mathrm{cm}$ raio inferior a $100 \mathrm{~m}$ para o intcrior da mata. Ocos que retiveram água neste exame foram numerados e visitados sistematicamente durante 12 meses e com intervalo de tempo não uniforme para as seis localidades.

O exame de cada oco para a determinação da infestação por mosquito Culicidae foi feito sifonando a água nele contida através de auxílio de bomba de sucção controlada manualmente (Fig. 2). Para certificar-se do número de formas imaturas existentes no microhabitat, foram leitas duas lavagens seguidas à retirada da água original, com água destilada, segundo técnica preconizada por Lounibos e col. ${ }^{8}$ (1981). Após contagem de cada estágio de desenvolvimento das formas imaturas ou exame negativo, as larvas e a primcira água fo- ram retornadas ao oco. Antes porćm, foi retirada amostra de larva não superior a $5 \%$ do número existente, para que fosse confirmado no laboratório a identificação feila no campo.

Nas duas chácaras foram colocados internódios de bambu para verificar se Ae. albopictus iria utilizá-los como criadouros. Dez unidades foram utilizadas, sendo que 5 tinham $20 \mathrm{~cm}$ de profundidade e outros $5 \mathrm{com} 40 \mathrm{~cm}$. Todos eram abertos e tinham diâmetro da boca correspondente a $10,5 \mathrm{~cm}$. Nos menores foi mantido volume de $500 \mathrm{ml}$ e nos maiores $1.000 \mathrm{ml}$. A cada exame desses microhabitals, o volume foi quase sempre corrigido e, na conduta para cxame larvário, seguiu-se o mesmo procedimento empregado nos ocos de árvores.

$\mathrm{Na}$ área urbana a pesquisa de formas imaturas ocorreu nos ocos das árvores e artefatos antrópicos de diversa natureza. O material encontrado foi todo transportado para o laboratório.

O ritmo de atividade levado a efeito nas seis localidades foi de 4.6 dias para as chácaras, quinzenais para as matas e alamedas e mensais para a árca urbana.

Para o cálculo da produtividade sazonal ou mensal de formas imaluras foram escolhidos cinco ocos, os quais foram responsávcis pela produção de aproximadamente 70,5 e $88,2 \%$, respectivamente de larva $\left(\mathrm{L}_{1}\right)$ e pupa. O rendimento desses indivíduos por colcta foi calculado através da média geométrica de Williams, transformadas

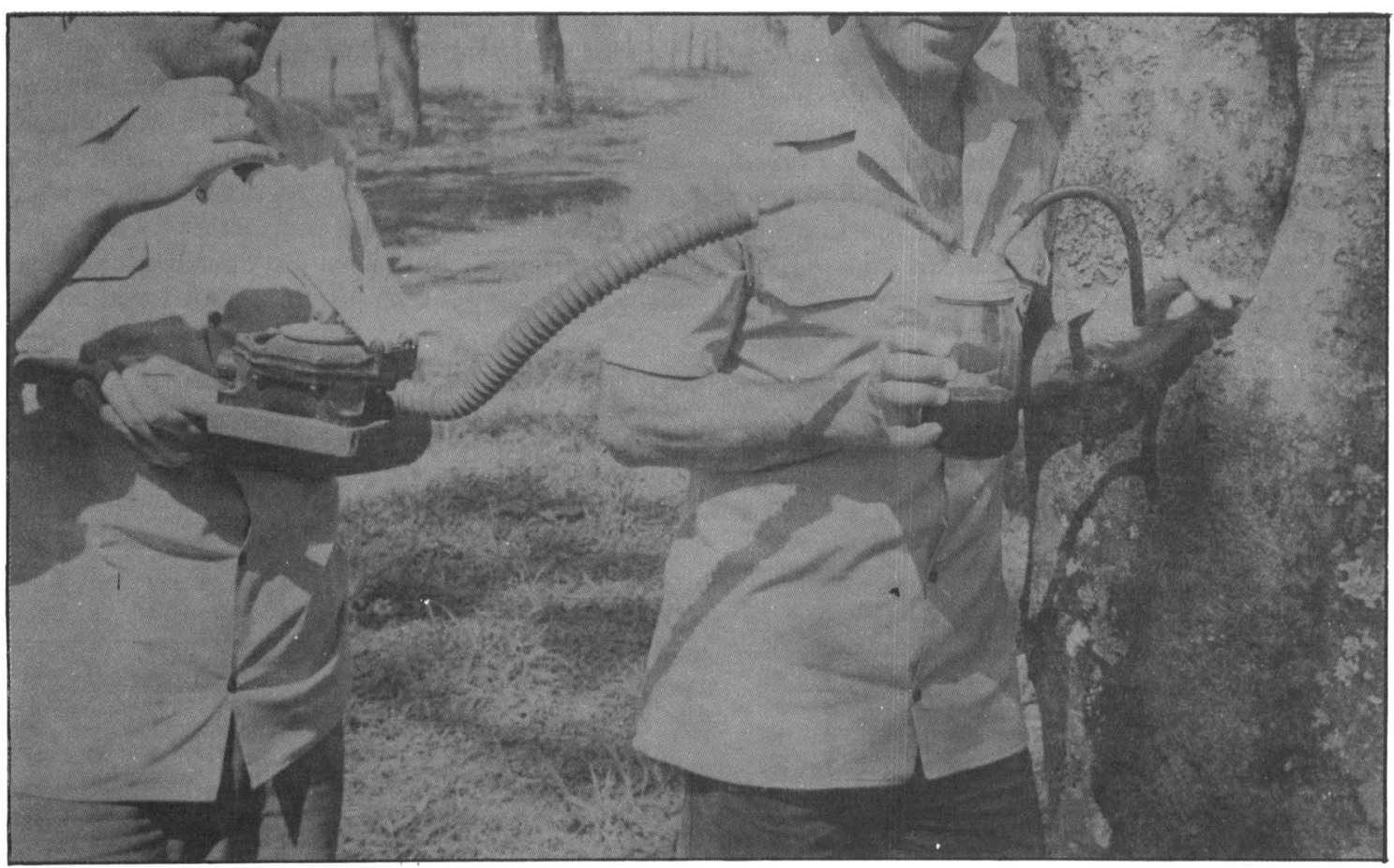

Figura 2. Extração de água dos microhabitats naturais. 
em porcentagem (Forattini e col. ${ }^{4}, 1981$ ). O índice de Breteau utilizado no presente estudo foi fornecido pela SUCEN-Regional Taubaté. Os índices pluviométricos e as temperaturas médias mensais foram fornecidas pclo Departamento de Águas e Energia Elétrica (DAEE), estação climática de Pindamonhangaba, situada a $12-15 \mathrm{~km}$ de distância dos pontos investigados.

\section{Resultados}

Microhabitats naturais: Foram catalogados 90 ocos de árvores e investigados sistematicamente durante um ano. Mostraram-se muito heterogêneos e, para facilitar a análise qualitativa e quantitativa da produção de larvas e pupas, foram agrupados em três categorias (Tabela 1). De forma geral, esses microhabitats foram relativamente pequenos, pouco freqüentes, e muitos recebendo insolação direta (Figs. 3A e B). Predominou o tipo aberto, formado pela bifurcação do caule e sujeito ao acúmulo de folhas mortas (Fig. 3A). A decomposição dessas folhas, provocada por microorganismos diversos, e a presença de formas imaturas de insetos não Culicidae produziram, muitas vezes, cheiro fétido na água do criadouro, particularmente quando as chuvas foram bem espaçadas.

Formas imaturas de Culicidae: Foram identificadas larvas e pupas de culicídeos em todos os microhabitats examinados, pertencentes às seguintes espécies: Ae. albopictus, Ae. terrens, Culex quinquefasciatus, $C x$. dolosus, Haemagogus leucocelaenus, $C x$. irridiscens e Toxorhynchites sp. Ae. albopictus foi assinalado em oco de árvore dos seguintes gêneros: Delonix (llambuaiã), Persea (abacateiro), Tibouchina (quaresmeira), Bauhinia (mão de vaca), Cassia (Cigarrcira), Clitoria e Stillingia. Na Tabela 2 constam os locais onde Ae. albopictus foi encontrado nos respectivos micro-

Tabela 1. Agrupamento dos ocos de árvores em categorias, segundo a capacidade volumétrica e profundidade, considerados microhabitats naturais potenciais de mosquito Culicidae, Tremembé, Vale do Paraíba, julho/89 a junho/90.

\begin{tabular}{crcrrrrrrr}
\hline & \multicolumn{3}{c}{ Volume medio em ml } & \multicolumn{4}{c}{ Profundidade } \\
Local & $>200$ & $200-600$ & $<600$ & Total & $>7$ & $7-15$ & $<15$ & Total \\
\hline A & 0 & 3 & 2 & 5 & 3 & 4 & 1 & 5 \\
B & 5 & 6 & 10 & 21 & 4 & 9 & 8 & 21 \\
C & 16 & 3 & 0 & 19 & 13 & 6 & 0 & 19 \\
D & 4 & 6 & 12 & 22 & 6 & 15 & 1 & 22 \\
E & 3 & 4 & 2 & 9 & 1 & 6 & 2 & 9 \\
F & 8 & 2 & 4 & 14 & 7 & 3 & 4 & 14 \\
\hline Total & 36 & 24 & 30 & 90 & 31 & 43 & 16 & 90 \\
\hline
\end{tabular}

habitats pesquisados. Neste particular, os microhabitats existentes na cidade e chácaras (Locais A e B) tiveram a colonização dessa espécie em nivel de até $100 \%$. No geral, Ae. albopicıus esteve presente uma ou mais vezes em cerca de $1 / 3$ dos microhabitats naturais pesquisados. Quanto às categorias aqui consideradas, a Tabela 2 registra que a maior positividade de Ae. albopictus ocorreu em ocos com volume superior a $600 \mathrm{ml}$ e profundidade entre $7-15 \mathrm{~cm}$. Contudo, a espécie foi capaz de colonizar todos os tipos, desde que contivessem água por algum tempo, curto ou prolongado. $\mathrm{O}$ primeiro caso, refere-sc à presença de larvas em brácteas de palmeira caídas ao solo.

Composição nos microhabitats: Ae. albopictus foi habitante solitário na maioria dos ocos existentes nos locais $A$ e $B$, enquanto que no $D$ (Alameda) esteve sempre associado a Ae. terrens (Tabela 3). Em 19 microhabitats naturais investigados na mata secundária (Local C), apenas em quatro deles situados no ecótono e com número reduzido de larvas foi constatada sua presença. Nas malas primárias $(E, F)$ esta espécie não foi detectada. Nesses dois ambientes verificou-se as seguintes composições: Ae. terrens/Sabethes purpureus; Ae. terrens/Cx. dolosus; Ae. terrens/Toxorhynchites sp; Ae. terrens/ Hg. leucocelaenus/Toxorhynchites sp. e Hg. leucocelaenus/Toxorhynchites $\mathrm{sp}$. Os artefatos antrópicos das chácaras e da área urbana foram os únicos com presença de formas imaturas de Ae. albopictus e $C x$. quinquefasciatus. Esses microhabitats foram $\mathrm{em}$ número e tipo extremamente diversificados, conforme descrição da PAHO ${ }^{16}$ (1987).

Composiçâo nos bambus: formas imaturas de $A e$. albopictus e $C x$. quinquefasciatus foram assinaladas nesse tipo de microhabitat (Tabela 3). A primeira espécie foi mais regularmente presente nas coletas, e somente em raras vezes foi constatada a ocorrência simultûnea das duas espécies (Tabela 3). O bambu com $40 \mathrm{~cm}$ e com $1.000 \mathrm{ml}$ foi

Tabela 2. Resultado das categorias de microhabitats naturais utilizados por $\mathrm{Ae}$. albopictus, segundo a capacidade volumétrica, Tremembé, Vale do Paraiba, junho/ 1989 a julho/1990.

\begin{tabular}{cccccccccc}
\hline & \multicolumn{3}{c}{ Volume em ml } & \multicolumn{4}{c}{ Profundidade } \\
Local & $<200$ & $200-600$ & $>600$ & Total & $<7$ & $7-15$ & 15 & Total \\
\hline A & 0 & 1 & 2 & 3 & 0 & 3 & 0 & 3 \\
B & 5 & $6^{*}$ & 10 & 21 & 4 & 9 & 8 & 21 \\
C & 2 & 1 & 0 & 3 & 2 & 1 & 0 & 3 \\
D & $1^{* *}$ & 0 & $4^{* *}$ & 5 & 1 & 4 & 0 & 5 \\
\hline Total & 8 & 8 & 16 & 32 & 7 & 17 & 8 & 32 \\
\hline
\end{tabular}

- Um criadouro associado com Culex quinquefasciatus

* Todos os criadouros associados com Aedes terrens 


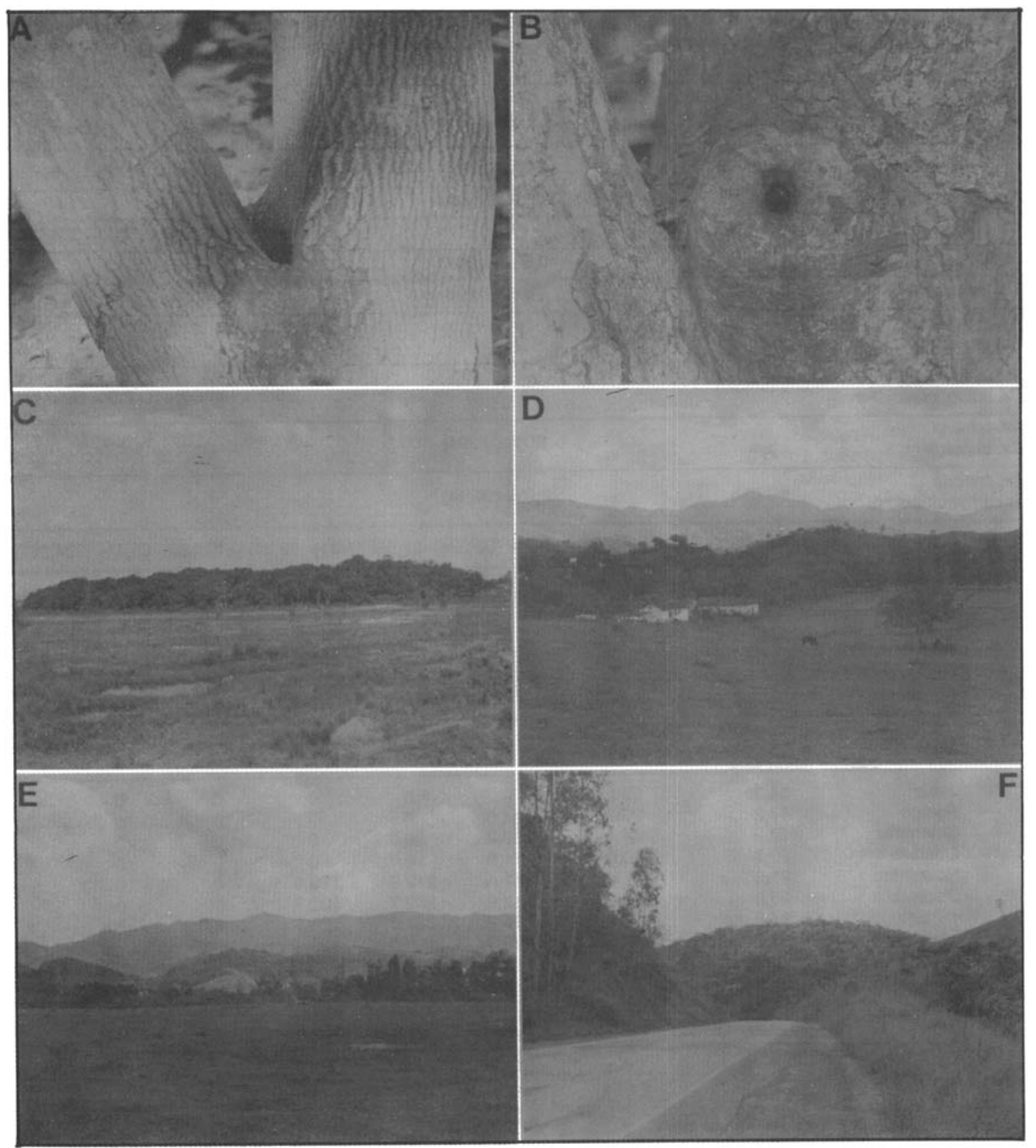

Figura 3. Microhabitats e macroambientes pesquisados. A-oco tipo aberto; B-oco tipo fechado; C-mata-secundária; Dalameda; E e F-mata residual primária.

proporcionalmentc o microhabitat mais procurado do que o de $20 \mathrm{~cm}$.

Condições climáticas: as condições climáticas representadas pela temperatura média do macrohabitat, chuva e sua influência no volume de água estocado em cinco ocos de árvores, constam da Tabela 4.

Durante o periodo das chuvas foi comum o encontro de até $100 \%$ dos ocos contendo água. Fora desse periodo, os microhabitats foram gradativamente secando. Entretanto, mesmo com a redução dos índices pluviométricos, alguns ocos retiveram água de chuva $\mathrm{em}$ volume suficiente para garantir o desenvolvimento de Ae albopictus. Assim sendo, e também $\mathrm{cm}$ função da constituição individual de cada um, os microhabitats alternaram-se entre secos e contendo algum volume de água, particularmente, no período de julho a novembro 
Tabela 3. Distribuição proporcional da composição especlfica de mosquito Culicidae em microhabitats naturais e experimentais, segundo quatro tipos de macrohabitats pesquisados, Tremembe, Vale do Paraiba, julho/1989 a junho/1990.

\begin{tabular}{|c|c|c|c|c|c|c|}
\hline \multirow[b]{2}{*}{ Especifica } & \multicolumn{4}{|c|}{ Ocos de Árvores } & \multicolumn{2}{|c|}{ Bambu } \\
\hline & Chácaras & Alameda & Matas & Cidade & $20 \mathrm{~cm}$ & $40 \mathrm{~cm}$ \\
\hline Ae. albopictus & $74,5 \%(507 / 680)$ & & $1,4 \%(4 / 294)^{*}$ & $22,5 \%(7 / 31)$ & $57,7 \%(208 / 360)$ & $62,5 \%(226 / 360)$ \\
\hline Ae. terrens & & $43,6 \%(61 / 140)$ & $22,1 \%(65 / 294)$ & & & \\
\hline Cx. quinquefasciatus & $0,3 \%(2 / 680)$ & & & & $0,3 \%(1 / 360)$ & $1,4 \%(5 / 360)$ \\
\hline Cx. dolosus & & & $0,3 \%(1 / 294)$ & & & \\
\hline Toxorhynchites sp. & $0,1 \%(1 / 680)$ & & $2,4 \%(7 / 294)$ & & & \\
\hline $\begin{array}{l}\text { Ae. albopictus } \\
\text { Ae. terrens }\end{array}$ & & $6,4 \%(9 / 140)$ & & & & \\
\hline $\begin{array}{l}\text { Ae. albopictus } \\
C x \text {. quinquefasciatus }\end{array}$ & $0,6 \%(4 / 680)$ & & & & $0,3 \%(1 / 360)$ & $2,0 \%(7 / 360)$ \\
\hline $\begin{array}{l}\text { Ae. terrens } \\
\text { Cx. dolosus }\end{array}$ & & & $1,0 \%(3 / 294)$ & & & \\
\hline $\begin{array}{l}A e . \text { terrens } \\
\text { Toxorhynchites sp. }\end{array}$ & & & $2,0 \%(6 / 294)$ & & & \\
\hline Total & $75,6 \%(514 / 680)$ & $46,4 \%(65 / 140)$ & $29,2 \%(86 / 294)$ & $22,5 \%(7 / 31)$ & $58,3 \%(210 / 360)$ & $66,1 \%(238 / 360)$ \\
\hline
\end{tabular}

- Positivo apenas em local C

Tabela 4. Resultados da temperatura média, precipitação de chuvas no Município de Pindamonhangaba, Vale do Paraiba e volume médio de água assinalado nos cinco microhabitats mais produtivos de Ae. albopictus, Tremembé, periodo de julho/1989 a junho/1990.

\begin{tabular}{lccccccc}
\hline Mês & $\begin{array}{c}\text { Temperatura } \\
\text { média }\end{array}$ & $\begin{array}{c}\text { Precipitação } \\
\mathbf{m m}^{3 *}\end{array}$ & $\mathbf{1}$ & $\mathbf{2}$ & $\mathbf{3}$ & $\mathbf{3}$ & $\mathbf{5}$ \\
\hline Julho & 14,2 & 53,2 & 0 & 0 & 0 & 0 & 0 \\
Agosto & 17,2 & 20,7 & 24,6 & 0 & 0 & 0 & 0 \\
Setembro & 19,2 & 94,1 & 380,8 & 80,0 & 0 & 0 & 0 \\
Outubro & 20,3 & 10,9 & 11,8 & 8,0 & 0 & 0 & 0 \\
Novembro & 21,9 & 147,4 & 351,5 & 218,3 & 129,0 & 0 & 0 \\
Dezembro & 23,0 & 150,0 & 447,6 & 249,7 & 102,8 & 0 & 0 \\
Janeiro & 24,8 & 128,2 & 661,2 & 281,0 & 226,7 & 208,0 & 48,8 \\
Fevereiro & 23,8 & 156,2 & 328,3 & 199,0 & 133,3 & 95,2 & $\mathbf{3 7 , 5}$ \\
Março & 23,7 & 103,9 & 785,0 & 448,2 & 468,8 & 188,5 & 70,5 \\
Abril & 22,4 & 70,5 & 527,2 & 291,1 & 609,2 & 329,5 & 72,7 \\
Maio & 17,6 & 79,2 & 702,0 & 370,5 & 939,8 & 236,3 & 55,3 \\
Junho & 15,9 & 1,3 & 100,7 & 37,2 & 705,0 & 27,3 & 55,5 \\
\hline
\end{tabular}

- Volume em milimetro

** Volume em ml

(Tabela 4). Notou-se, ainda, que durante as chuvas mais pesadas e prolongadas houve aumento do número de criadouros ễêmcros de Ae. albopictus, como foi o caso de tocos de bambu, brácteas de palmeiras e pequenos recipientes artificiais. $\mathrm{Na}$ Tabela 4 também estão registradas as tempcraturas médias mensais do macroambiente.

Produtividade de formas imaturas: no periodo de janeiro a maio os microhabitats com volumes entre $200-600 \mathrm{ml}$ foram positivos com até 100 formas imaturas por coleta, enquanto que os abaixos de $200 \mathrm{ml}$ raramente ultrapassavam o número de
10 indivíduos. Fora deste periodo, somente os primeiros foram capazes de apresentar positividade de 1 a 25 exemplares em apenas três oportunidades. Com relação aos criadouros de Ae. albopictus, com volume acima de $600 \mathrm{ml}$, observou-se que de janeiro a maio o rendimento da coleta/oco esteve nos limites de 50 a 900 formas imaturas, sendo que o número médio esteve em torno de 250. Esclarece-se que esses valores somente foram alcançados quando os níveis de água dos ocos não foram inferiores a $3 / 4$ de sua capacidade máxima, e que o número elcvado de formas imaturas se de- 
veu à predominância invariável de $\mathrm{L}_{1}$ e $\mathrm{L}_{2}$. Nos meses de julho a novembro a redução do número de forma imaturas foi extremamente elevada, mesmo que chuvas eventuais elevassem o nível de água dos microhabitats naturais.

A produção de formas imaturas para Ae. albopictus através do bambu experimental indicou haver sobreposição nos meses observados para os ocos das árvores. Embora o bambu de $1.000 \mathrm{ml}$ tivesse apresentado resultado absoluto supcrior ao de $500 \mathrm{ml}$, o número de indivíduos por coleta ficou entre 200 e 300 . Já no período de julho a novembro, a positividade foi assinalada apenas três vezes, com valores de 4,15 e 22 formas imaturas.

A freqüência sazonal calculada apenas para o período de dezembro a junho e a referente às larvas de primeiro estádio $\left(\mathrm{L}_{1}\right)$ e pupas constam da Figura 3. Assim sendo, a média de Williams para Ae. albopictus foi calculada a partir dos resultados obtidos nos cinco microhabitats mais procurados pelo mosquito.

Finalmente, a espécie predominante no ambiente natural foi Ae. terrens e sua maior freqüência ocorreu nos meses de janeiro a maio. Foram porém registrados encontros esporádicos nos meses de julho, agosto e setembro.

\section{Discussão}

$\mathrm{Na}$ faixa tropical terrestre existem várias espécies de mosquito Culicidae cujos estágios imaturos ocupam ocos de árvore (Foratlini ${ }^{3}, 1962$ e Lounibos e col. $\left.{ }^{8}, 1981\right)$. Ae. albopictus é notável habitante desses microhabitats e também de inúmeros artefatos antrópicos (Hawley ${ }^{7}, 1988$ ).

No Brasil, os estudos sobre a comunidade de ocos de árvore têm sido muito negligenciados. Porém, como houve interesse $\mathrm{cm}$ conhecer os microhabitats de Ae. albopictus na região do Vale do Paraíba e as inter-relações desta espécie com outras autóctones, que dividem igualmente o mesmo nivel trófico, tornou-se possivel explicitar algumas informaçōes sobre $o$ assunto.

Levando-se em conta a segregação espacial de culicídcos nas áreas estudadas, verificou-se a presença de sete populações Culicidae, sendo que o gênero Aedes esteve presente apcnas com Ae. terrens e Ae. albopictus. Essa referida segregação se deveu às preferências para tipo de macro e microhabitats, pois as espécies autóctones foram assinaladas quase que exclusivamente no ambiente florestal primário. No caso do Ae. terrens, seu raio de ação se estendeu até a zona rural (Locais C e D). Neste particular, o encontro anterior foi relatado por Neves e Faria ${ }^{15}$ (1977) em ocos de árvore das matas periféricas de Belo Horizontc. Ae. albopic- tus preferiu colonizar microhabitats existentes nas chácaras e na cidade (Tabcla 2), porém em baixa frequiência e número restrito de indivíduos que alcançaram a zona rural (Locais C e D). Tais achados concordam com os dados da literatura, sobretudo no que diz respeito a sua maior preferência por área rural-urbana ou suburbana (Hawley ${ }^{7}, 1988$ ).

Foram observadas associaçðes interespecíficas de Ae. albopictus com espécies autóctones (Tabela 3). Porém, essa espécie foi predominantemente habitante solitário dos microhabitats naturais das chácaras e da cidade, com 74,5 e $22,5 \%$ de positividade por coleta, respectivamente. A exceção ocorreu na Alameda (Local D) onde esteve sempre associada com Ae. lerrens em 6,4\% das coletas (Tabela 3). Não obstante a ocorrência desse fato, os baixos valores de sobreposição aqui observados sugerem a quase inexistência de competidores. Sua associação com Ae. terrens não evidenciou caráter de compctição substitutiva. "A priori", essa cohabitação pareceu ser similar à verificada entre Aedes triseriatus e Ae. albopictus em Louisiana, EUA (Schreiber e col. ${ }^{19}, 1988$ ). Por outro lado, o desaparecimento da discreta infestação de Ae. aegypti na rcgião, após detecção de Ae. albopictus, não foi investigada, mas levando-se em conta que a substituição daquele mosquito por este, em algumas localidades americanas ter sido atribuída, em parte, à melhor adaptação de Ae. albopictus (Moore e col. $\left.{ }^{12}, 1988\right)$, pode-se analogamente admitir que este aspecto deva estar ocorrendo na região estudada. Na Tailândia, Ae. albopictus foi encontrado associado com Aedes seatoi (Harrison e col. ${ }^{6}, 1972$ ). A PAHO ${ }^{16}$ (1987) cita vários relatos similares na Ásia cujas populações implicadas foram, Ae. malayensis, Ae. pseudoalbopictus, Ae. japoniensis e Ae. logoi.

Na região do Vale do Paraíba, a infestação de Ae. albopictus guardou relação direta com a atividade antrópica, uma vez que a comunidade Culicidae de ocos de árvore foi praticamente eliminada. Daí, resultando a vacância de nichos ecológicos naturais. Assim sendo, se Ae. albopictus não está encontrando compctidores autóctones o processo para sua adaptação tornou-se mais favorccido, quer pelas condiçōes ccológicas referidas quer pela sua flexibilidade genética (Rail ${ }^{17}, 1986$ ). Ecologicamentc, isto significa admitir que a infestação desse mosquito no Vale do Paraíba ocorre sem obrigatoricdade de novas inter-rclações. Essa feição, em parte, parece justificar a rápida disseminação de Ae. albopictus no Estado de São Paulo, conforme relato de Buralli e $\mathrm{col}^{2}$. (1991). Por outro lado, sua ausência no ambiente florestal primário poderá ser questão de tempo ou de barreiras naturais ainda não vencidas.

Não obstante o encontro de formas imaturas de 
Ae. albopictus em gama diversificada de microhabitats naturais, os ocos com volumes superiores a $600 \mathrm{ml}$ e profundidades entre 7 e $15 \mathrm{~cm}$ foram os preferidos (Tabcla 2). Aliás, a maior produção de larvas e pupas nesses criadouros sugere ter havido várias oviposições, sobretudo, por cclosõcs que possibilitaram a coleta, por vezes, de até 900 larvas de primeiro estádio. Tal caraclerística é compalível com o padrão Stegomyia cujas cclosões ao primeiro estímulo podem alcançar taxas de 50 a 95\% (Lounibos e col. ${ }^{8}, 1981$ ). Além disso, esta condição favorece também a existência de criadouros com baixa estocagem de água, como aqueles com volumes até $200 \mathrm{ml}$ (Tabela 2). Daí a razão pela qual Lounibos e col. ${ }^{8}$ (1981) consideraram que a ocupação de ocos de árvores, com grandes volumes "versus" pequenos, não estão necessariamente correlacionados com qualquer padrão de eclosão. Sinsko e Craig ${ }^{21}$ (1981) observaram que a produtividade de $23 \%$ dos ocos corresponderam a $83 \%$ de pupa de Ae. triseriatus, enquanto que Yates ${ }^{22}$ (1979) observou que o número de adultos emergentes de Aedes geniculatus foi obtido em criadouro com grande volume de água. Lounibos e col. ${ }^{8}$ (1981) verificaram que a cepa silvestre de Ae. aegypti de Makabara (África) é favorecida pelos grandes ocos, Mori'13 (1979) notou que ovos de Ae. albopictus eclodem simultancamente após chuva e, por vezes, mais de 600 larvas puderam ser vistas em garrafas de $500 \mathrm{ml}$. No presente estudo, apenas $17,7 \%$ dos ocos que tinham volumes superiores a $600 \mathrm{ml}$ c limite máximo de $5.455 \mathrm{ml}$, foram assinalados com larvas e pupas de Ae. albopictus (Tabela 2). O resultado obtido com internódio de bambu com volumes de $500 \mathrm{e}$ $1.000 \mathrm{ml}$ evidenciaram taxas de positividade de 57,7 e $62,5 \%$, respectivamente. Dessa forma, esta informação complementar parece confirmar tendência da espécie para microhabitats com grandes volumes de água, particularmente pela condição favorável à manutenção de água por período de tempo mais prolongado. Ademais, a oviposição em microhabitats com baixa chance de produzir adultos poderá significar caractcrística adicional de excelente invasor.

De maneira geral, foi coincidente o padrão de abundância estacional de culicídeos cm microhabitats naturais com a época das chuvas (Tabela 4 e Fig. 4). Porém, os meses de chuvas mais pesadas não foram simultâneos com o período produtivo mais significativo para Ae. albopictus. E, isso porque, como a Figura 4 mostra que as eclosocs tiveram início no mês de dezembro co pico máximo de larvas e pupas no período de março-abril, quando 0 índice pluviomérrico no mês de abril correspondeu à metade da ocorrida em fevereiro (Tabela 4). De julho a novembro, larvas de Ae. albopiclus for-
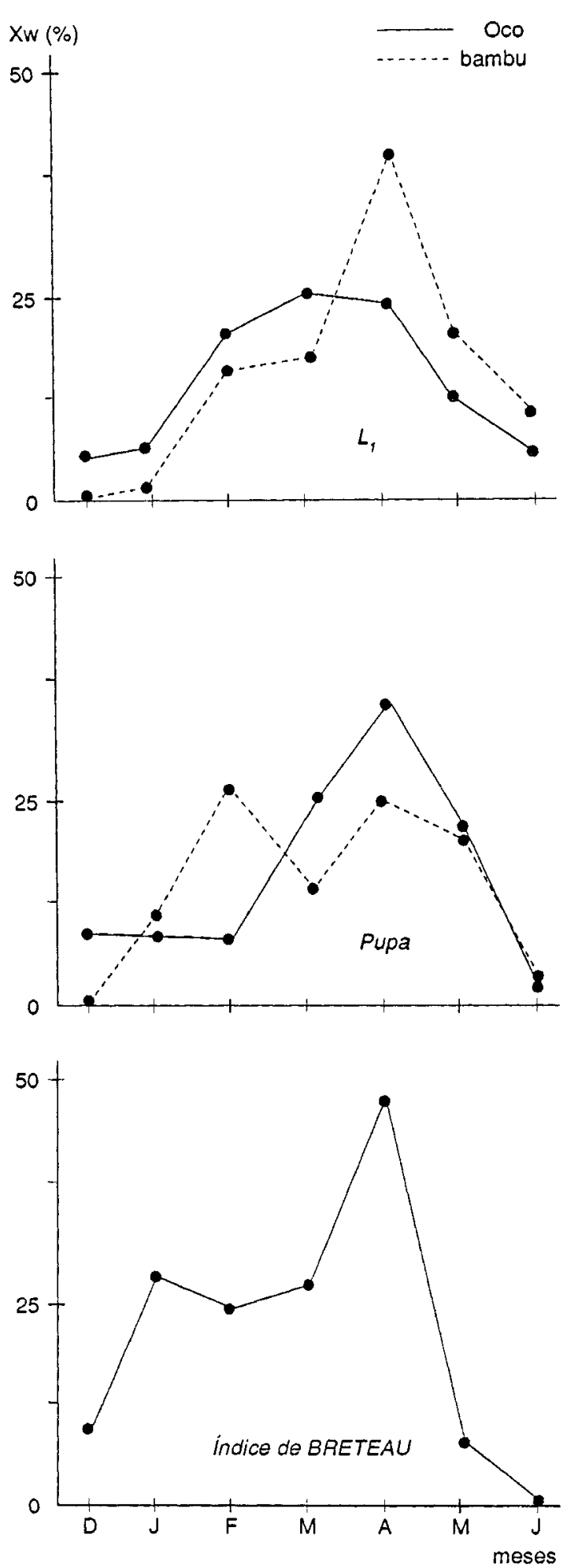

Figura 4. Densidade mensal de larvas de primeiro estádio e pupa de Aedes albopictus em microhabitats naturais, bambus experimentais e artefatos antrópicos, Tremembé, Vale do Paraiba, 1989/90. 
am coletadas em quantidade e $\mathrm{cm}$ número reduzido de $\propto$ cos. Comparativamente, o resultado do bambu experimental foi semelhante, exceto quanto ao pico isolado de pupa no mês de feverciro, quando ocorreu maior índice pluviométrico, i. é., $156,2 \mathrm{~mm}$. Sinsko e Craig ${ }^{21}$ (1981) observaram que as chuvas pesadas influenciaram diretamente na produção de pupas de Ae. triseriatus, o que coincide parcialmente com o comportamento da cepa paulista de Ae. albopictus. Assim sendo, os resultados obtidos no presente trabalho confirmam a cstação vcrão $c$ outono como periodos de "input" de larvas e pupas. Da mesma forma, os resultados conseguidos no ambiente urbano atsavés do Índice de Breteau e nos presentes resultados oblidos foram sazonalmente simultâneos (Fig. 4 ). Em Singapura, Ae. albopictus apresentou três picos estacionais, ou scja, dezcrnbro, abril-maio e agosto-setembro (PAHO ${ }^{16}, 1987$ ). Porlanto, é lícilo pensar que esse comportamento padrão apresentado pela cepa paulista, embora com divergência sazonal, sugira origem da Ásia tropical.

Quanto à relação da temperatura média do macroambiente com o desenvolvimento larvário, verificou-se que o aparecimento desses indivíduos teve início a $23^{\circ} \mathrm{C}$, mas com signilicativo desenvolvimento até $17^{\circ} \mathrm{C}$. Igualmente ao ocorrido com as chuvas, a estação verão, que foi a mais quente, produziu menor número de larvas do que o outono (Tabela 4 e Fig. 4). Portanto, a faixa mais adequada ao desenvolvimento larvário pareceu situar-se nos limites de 23 a $17^{\circ} \mathrm{C}$. Tal resultado é oposto ao verificado para cepas com distribuição no Hemisfério Norte (Nawrocki e Hawlcy ${ }^{14}$, 1987). Corrobora com aquela situação, as obscrvaçð̄es laboratoriais que revelaram a incapacidade aparente da cepa brasileira de Ae. albopictus para realizar diapausa (Craig Jr. apud Moore e col. ${ }^{12}, 1988$ ). Conseqüentemente, essas informações sugercm, taumbém que $A e$. albopictus do Vale do Paraíba scja cepa tipicamente tropical.

Do ponto de vista epidemiológico, a infestação e adaptação de $A e$. albopictus em distintos gradientes ecológicos situados no transecto estudado, assemelha-se ao observado no sudeste asiático. Por isso, há possibilidade desta espécie precncher elo necessário ao intercâmbio de agentes selváticos com o ambiente urbano $\mathrm{c}$ vice-versa ou scrvir de reservatório vertical de vírus imporlados, face às comprovações experimentais de Mitchell c Miller $^{10}, 1990$ e Rosen ${ }^{18}, 1987$. Por outro lado, essa infestação tambćm ocorre entre os dois maiores centros demográficos e cconômicos do Brasil, com intenso intercâmbio rodoviário, ferroviário e aéreo. Logo, a comprovação clínica de casos importados de dengue na região, não deixa de ser ameaça e, nesse sentido, são atuais as preocupaçōes referidas por Monath ${ }^{11}$ (1986).

\section{Conclusóes}

Diante das considerações e análise levadas a efeito neste trabalho pode ser possivel emitir as seguinics conclusões:

1. A cepa de Ae. albopiclus presente no Vale do Paraíba mostrou qualidade de excelcnte invasor, ao utilizar intensamentc a vacância de nichos ecológicos naturais, sem deixar de lado a ocupação de inúmcros outros de natureza antrópica.

2. A árca rural-urbana (chácaras) pareceu ser o ambientc ecológico preferido, scmclhantemente ao obscrvado no sudesie da Ásia.

3. A abundância de larvas e pupas ocorreu nas estaçōes verão e outono, com o pico máximo correspondente ao mês de abril.

4. O presente conhecimento conduz à suposição de que a cepa de Ae. albopictus presente no Vale do Paraíba, scja originária da Ásia tropical.

5. Epidemiologicamente, essa infestação representa impacto à saúde pública, face ao risco potencial de Ae. albopictus vir a excrcer, além do dengue, papel velorial para diversas outras arboviroses, tais como febre amarcla e outras, até então considerada exclusivamente selvática $\mathrm{cm}$ nosso meio.

\section{Agradecimentos}

À SUCEN pclas facilidades proporcionadas, sobretudo pela sua equipe de campo, e ao Sr. Daniel C. Flores, pela exccução do mapa da região estudada.

GOMES, A. de C. et al. [Microhabitats of Aedes albopictus (Skuse) in the Paraiba Valley region of the State of S. Paulo, Brazil]. Rev. Saúde públ., S. Paulo, 26: $108-18,1992$. The objective of this study was the determination of extent of the spacial distribution of Aedes albopictus in the Paraíba River Valley, State S. Paulo, Brazil. Thus, collections of larvae and pupae mosquitoes were carried out at six sites distributed along a transection with a $10-\mathrm{km}$ extension. The target was the treeholes but artificial containers were also used in this investigation. Aedes terrens and Ae. albopictus were the only species of genus Aedes present in the treeholes mentioned. The segregation of seven species of the treehole community was undertaken in the light of macrohabitat and microhabitat features. Thus, the distribution of Ae. albopictus was found to cover the rural, nuralurban and urban zones, but the rural-urban held the prefcrence. Ae. albopictus never present in the residual and primary forest. The favorable factor to infestation with Ae. albopictus in the Paraíba Valley seems to have been the large number of natural niches made vacant by human influence. The rain has been important in the production of larvae and pupac, but the rainfall period does not coincide with the maximum production on them. 
The treeholes whose volume exceeded $600 \mathrm{ml}$ were the most productive breeding places. The abundance of these two stages occurs in the summer and autunm. However, the highest peak was observed in the months of March and April. These seasonal variations were found to be common in both the bamboo trap and the artificial container. The temperature data suggest a limit of from 17 to $23^{\circ} \mathrm{C}$ for the best development of larvae. In the light of this, the strain of Ae. albopictus studied seems to have originated in tropical Asia. Just as happaned with $A e$. aegypti it may become an important epidemiological vector for the dengue fever and provide links for yellow fever transmission in Brazil.

\section{Keywords: Aedes. Ecology, vectors.}

\section{Referências Bibliográficas}

1. BRTTO, M.; MARQUES, G. R. A. M.; MARQUES, C. A. TUBAKI, R. M. Primeiro encontro de Aedes (Stegomyia) albopictus no Estado de São Paulo (Brasil). Rev. Saúde públ., S. Paulo, 20: 489, 1986.

2. BURALLI, G. M.; MARQUES, G. R. A. M.; GEROLA Jr., O. Distribuição geográfica de Aedes aegypti c Aedes albopictus no Estado de São Paulo. [Apresentado ao Congresso da Sociedade Brasileira de Medicina Tropical, 279. Uberaba, 1991 - Resumo].

3. FORATTINI, O. P. Entomologia médica. São Paulo, Ed. USP, 1962, v. 1.

4. FORATTINI, O. P.; GOMES, A. de C.; SANTOS, J. L. F.; GALATI, E. A. B.; RABELLO, E. X.; NATAL, D. Observações sobre atividade de mosquitos Culicidae, em mata residual no Vale do Ribeira, São Paulo, Brasil. Rev. Saúde públ., S. Paulo, 15: 557-86, 1981.

5. GOMES, A. de C. \& MARQUES, G. R. A. M. Encontro de criadouro natural de Aedes (Stegomyia) albopiclus (Skuse), Estado de São Paulo, Brasil. Rev. Saúde públ., S. Paulo, 22: 245, 1988.

6. HARRISON, B. A.; BOONYAKANIST, P.; MONGKOLPANYA, K. Biological observations on Aedes seatoi Huang in Thailand with notes on rural Aedes aegypti (L) and other Stegomyia populations. J. med. Ent., 9:1$16,1972$.

7. HAWLEY, W. A. The biology of Aedes albopictus. J. Amer. Mosquito Control Ass., 4: 2-39, 1988.

8. LOUNIBOS, L. P. Habitat scgregation among African trehole mosquitos. Ecol. Ent., 6: 129-54, 1981.

9. MARANGONE, A. B. \& ORTIZ, L. P. Região do Vale do
Paraíba: mudanças recentes em uma área de povoamento anligo. São Paulo, Fundação SEADE, 1985. (Série Análise Demográfica Regional 4).

10. MITCHELL, C. J. \& MILLER, B. R. Vertical transmission of dengue viruses by strains of Aedes albopictus recently introduced into Brazil. J. Amer. Mosquito Control Ass., 6: 251-3, 1990.

11. MONATH, T. P. Aedes albopictus, an exotic mosquito vector in the United State. Ann. intern. Med., 105: 449-51, 1986.

12. MOORE, C. G.; FRANCY, D. B.; ELIASON, R. E.; MONATH, T. P. Aedes albopictus in the United State: rapid spread of a potencial disease vector. J. Amer. Mosquito Control Ass., 4: 356.61, 1988.

13. MORI, A. Effects of larval density and nutrition on some atributes of immature and adult Aedes albopictus. Trop. Med., 21: 85-103, 1979.

14. NAWROCKI, S. J. \& HAWLEY, W. A. Estimation of the northem limits of distribution of Aedes albopictus in North America. J. Amer. Mosquito Conirol Ass., 3: 314-7, 1987.

15. NEVES, D. P. \& FARIA, A. C. Biologia do Aedes (Finlaya) terrens (Walker) em condições de campo e laboratório (Diptera, Culicidac). Rev. bras. Biol., 37: 803-6, 1977.

16. PAN AMERICAN HEALTII ORGANIZATION. Executive Committee of the Directing Council. Aedes albopictus in the Americas; $99^{\text {th }}$ meeting. Washington, D. C., 1987. (CE99 15) [Mimeografado].

17. RAI, K. S. Genetics of Aedes albopiclus. J. Amer. Mosquito Conirol Ass., 2: 429-36, 1986.

18. ROSEN, L. Sexual transmission of dengue viruses by Aedes albopictus. Amer J. trop. Med. Hyg., 37: 398$402,1987$.

19. SHREIBER, E. T.; MELK, C. L.; YATES, M. N. Verical distribution and species coexistence of treehole mosquitoes in Louisiana. J. Amer. Mosquito Control Ass., 4: 9 $13,1988$.

20. SECRLTARIA DE ESTADO DE ECONOMIA E PLANEJAMENTO. Plano regional do macroeixo paulista. São Paulo, 1978.

21. SINSKO, M. J. \& CRAIG Jr., G. B. Dynamics of an isolated population of Aedes triseriatus (Diptera: Culicidae). II - Factors affecting produtivity of immature stages. $J$. med. Ent., 18: 279-83, 1981.

22. YATLS, M. G. The biology of the treehole breeding mosquito Aedes geniculalus (Oliver) (Diptera: Culicidae) in southem England. Bull. ent. Res., 69: 611-28, 1979.

Recebido para publicaçäo em 26/8/1991 Aprovado para publicação em 26/3/1992 